\title{
Application of Segmentation Techniques on Atherosclerosis Images
}

\author{
Patel Janakkumar Baldevbhai ${ }^{1}$, R.S. Anand ${ }^{2}$ \\ ${ }^{I}$ (Research Scholar, Electrical Engineering Department, Indian Institute of Technology Roorkee, India) \\ ${ }_{2}^{2}$ (Professor, Electrical Engineering Department, Indian Institute of Technology Roorkee, India)
}

\begin{abstract}
This paper presents application of segmentation techniques on atherosclerosis images using various segmentation methods like Otsu thresholding, fuzzy C means, clustering algorithm and marker controlled watershed segmentation algorithm. Atherosclerosis is one of the causes of coronary heart disease $(C H D)$. The proposed marker controlled watershed algorithm for medical image segmentation and analysis is very important because of its advantages, such as always being able to construct an entire division of the color image and prevent over segmentation as compared to conventional watershed algorithm. Paper finds Region of Interest (ROI) values of segmented image with proposed technique for coronary atherosclerosis.
\end{abstract}

Keywords - Coronary heart disease (CHD), Clustering Image Segmentation Technique, Medical Image Segmentation, Fuzzy C means algorithm, Marker based Watershed Image Segmentation Technique.

\section{Introduction}

Image segmentation is a vital method for most medical image analysis tasks. Segmentation is an important process to extract information from complex medical images. Image segmentation is a necessary preliminary step for any image analysis task. This process partitions an image into a number of constituting regions. Each partition region is homogeneous with respect to a given property, while the set including any two adjacent regions is not homogeneous. Watershed transformation (WT) [1] is a basic tool for image segmentation exploiting both region-based and edge-detection-based methodologies. Segmentation is accomplished by using the watershed [2] transformation, which provides a partition of the image into regions whose contours closely fit. The watershed transformation considers the gradient magnitude of an image as a topographic surface. Pixels having the highest gradient magnitude intensities (GMIs) correspond to watershed lines, which represent the region boundaries. Water placed on any pixel enclosed by a common watershed line flows downhill to a common local intensity minimum (LIM). Pixels draining to a common minimum form a catch basin, which represents a segment. Luc Vincent [3] presented a morphological grayscale reconstruction algorithm for image analysis. Serge Beucher [4] redefined hierarchical segmentation by means of a new algorithm called the waterfall algorithm. V. Grau [5] et al. presented an improvement to the watershed transform that enables the introduction of prior information in its calculation with the information via the use of a previous probability calculation. Hardening of the arteries, also called atherosclerosis, is a common disorder. It occurs when fat, cholesterol, and other substances build up in the walls of arteries and form hard structures called plaques. Over the course of years and decades, plaque build-up narrows patient's arteries and makes them stiffer. These changes make it harder for blood to flow through them. Coronary heart disease (CHD) is a narrowing of the small blood vessels that supply blood and oxygen to the heart. CHD is also called coronary artery disease. Coronary heart disease (CHD) is the leading cause of death in the United States for men and women. Coronary heart disease is caused by the build-up of plaque in the arteries to patient's heart. This may also be called hardening of the arteries. Atherosclerosis is a disease characterized by a deposit of plaque in an arterial wall over time. The disruption of an atherosclerotic plaque is considered to be the most frequent cause of heart attack and sudden cardiac death. Studying vulnerable plaques constitutes a major research area in the field of clinical and medical imaging. In order to track progression and regression during therapy of the atherosclerosis, the inner and outer borders of the arterial wall are extracted and the plaque area is identified in the region between these two borders. Atherosclerosis is an underlying cause of cardiovascular disease, which impacts the health of more than $30 \%$ of the U.S. population. Valerie Pazos et al. [6] presented research on mechanical characterization of atherosclerotic arteries. Carotid atherosclerosis is a primary cause of transient ischemic attack and stroke. Accumulating evidence suggests that it is most likely not the size of the carotid atherosclerotic plaque nor the per cent stenosis of the carotid lumen that determines the risk for stroke. Rather plaque composition and activity are now thought to play central roles in determining the severity of the disease and the probability for plaque rupture [7]. Danijela $e t$ al. presented a method for carotid artery vessel wall segmentation in computed tomography angiography (CTA) data [8]. Gozde Unal et al. presented a shape-driven approach to segmentation of the arterial wall from intravascular ultrasound images in the rectangular domain [9]. Simon et 
al. [10] presented a research paper on vulnerable atherosclerotic plaque elasticity reconstruction based on a segmentation-driven optimization procedure using strain measurements a theoretical framework approach.

Definition: Atherosclerosis is defined by the WHO as variable combination of focal accumulation of lipids, complex carbohydrates, blood and its constituents, fibrous tissue and calcium deposits combined with its changes of the media.

Atherosclerosis is thus a patchy, nodular type of arteriosclerosis. It is a process of hardening of the arteries [41]. A catheter is inserted (figure 5) through the patient's groin into an artery and pushed toward the distal end of the coronary arteries. Thereafter, the ultrasound transducer in the catheter is pulled back with constant speed. During the pullback, sequences of images are acquired. The lumen is the interior of the vessel, through which the blood flows. The intima is the innermost layer of an artery. It is made up of one layer of endothelial cells and is supported by an internal elastic intima. The endothelial cells are in direct contact with the blood flow. It is a fine, transparent, colorless structure that is highly elastic. The media is the middle layer of an artery, which is made up of smooth muscle cells and elastic tissue. The adventitia is the outermost layer of the blood vessel, surrounding the media. It is mainly composed of collagen. Extraction of the boundaries of the coronary arterial wall by segmenting the lumen and media adventitia contours is a first step in measuring quantities such as lumen diameter and plaque dimensions, and assessment of the atherosclerotic plaque. Manual segmentation and processing of the lumen contour and the media-adventitia contour is tedious, time-consuming, and susceptible to intra- and interobserver variability. Due to the high number of images, typically in the order of hundreds, automated segmentation of the arterial contours is an essential task.

Image segmentation algorithms are classified into two types, supervised and unsupervised. Unsupervised algorithms are fully automatic and partition the regions in feature space with high density. The different unsupervised algorithms are Feature-Space Based Techniques, Clustering (K-means algorithm, Cmeans algorithm), Histogram thresholding, Image-Domain or Region Based Techniques (Split-and-merge techniques, Region growing techniques, Neural network based techniques, Edge Detection Technique), Fuzzy Techniques. The watershed segmentation technique has been widely used in medical image segmentation. Watershed transform is used to segment medical images. The method originated from mathematical morphology that deals with the topographic representation of an image. Neural Network based algorithm like Pulse Coupled Neural Network (PCNN) can be utilized as a supervised algorithm for image segmentation.

Segmentation is often a critical step in image analysis. Microscope image components show great variability of shapes, sizes, intensities and textures. An inaccurate segmentation conditions the ulterior quantification and parameter measurement. The Watershed Transform is able to distinguish extremely complex objects and is easily adaptable to various kinds of images. The success of the Watershed Transform depends essentially on the existence of unequivocal markers for each of the objects of interest. The standard methods of marker detection are highly specific, they have a high computational cost and they determine markers in an effective but not automatic way when processing highly textured images. This paper implements segmentation techniques using Otsu thresholding method, Fuzzy $\mathrm{C}$ means level 0 and level 1 method, color clustering technique and proposed marker controlled watershed segmentation technique on atherosclerosis images. This proposed marker controlled watershed algorithm for medical image segmentation and analysis is very important and useful because of its advantages, such as always being able to construct an entire division of the color image and prevent over segmentation as compared to conventional watershed algorithm.

Watersheds are one of the typical regions in the field of topography. A drop of the water falling it flows down until it reaches the bottom of the region. Monochrome image is considered to be a height surface in which high-altitude pixels correspond to ridges and low altitude pixels correspond to valleys. This suggestion says if we have a minima point, by falling water, region and the frontier can be achieved. Watershed uses image gradient to initial point and region can get by region growing. The accretion of water in the neighbourhood of local minima is called a catchment basin. Watershed refers to a ridge that divides areas shattered by different river systems. A catchment basin is the environmental area draining into a river or reservoir. If we consider that bright areas are high and dark areas are low, then it might look like the plane. With planes, it is natural to think in terms of catchment basins and watershed lines.

An intangible water drop is placed at each untagged pixel. The drop moves to low amplitude neighbour until it reaches a tagged pixel and it assumes tag value. In flooding approach, intangible pixel holes are pierced at each local minima. The water enters the holes and takings to fill each catchment basin. If the basin is about to overflow, a dam is built on its neighbouring ridge line to the height of high altitude ridge point. These dam borders correspond to the watershed lines. Advantages of the watershed transform include the fact that it is a fast, simple and intuitive method. More importantly, it is able to produce an entire division of the image in separated regions even if the contrast is poor, thus there is no need to carry out any post processing work, such as contour joining. Its limitations will include over-segmentation and sensitivity to noise. There has also been an increasing interest in applying soft segmentation algorithms, where a pixel may be classified partially into multiple classes, for medical images segmentation. Clustering is a method of grouping a set of patterns into a 
number of clusters such that similar patterns are assigned to one cluster. Each pattern can be represented by a vector having many attributes. Clustering technique is based on the computation of a measure of similarity or distance between the respective patterns. A cluster is a collection of objects which are similar between them and are dissimilar to the objects belonging to other clusters. Unlike classification, in which objects are assigned to predefined classes, clustering does not have any predefined classes. The main advantage of clustering is that interesting patterns and structures can be found directly from very large data sets with little or none of the background knowledge. The cluster results are subjective and implementation dependent. K-means clustering is a simple clustering method with low computational complexity. The clusters produced by K means clustering do not overlap. The Fuzzy C-means clustering algorithm is a soft segmentation method that has been used extensively for segmentation of medical images. In this work, we use K-means clustering and Fuzzy C-means Clustering methods exclusively to produce a segmentation of the image. In this research paper we implemented color clustering algorithm, Fuzzy C Means clustering as well as marker controlled watershed segmentation algorithm separately for medical image segmentation. The Clustering algorithms are unsupervised learning algorithms, while the marker controlled watershed segmentation algorithm makes use of automated thresholding on the gradient magnitude map and post-segmentation merging on the initial partitions to reduce the number of false edges and over-segmentation.

Segmentation has wide application in medical field [11-15]. Having good segmentations will help clinicians and patients as they provide vital information for 3-D visualization, surgical planning and early disease recognition. Microscope image components show great variability of shapes, sizes, intensities and textures [16]. Moreover, during acquisition, it is necessary to establish a high number of parameters that result in the presence of noise, non-homogeneous illumination, fuzzy contours and low contrast. This characteristic results in an incorrect segmentation when applying conventional segmentation methods. Watershed Transform (WT) is a powerful morphological tool to segment texture images into regions of interest. This transform is adaptable to different types of images and capable of distinguishing extremely complex objects. The WT is a segmentation method based on regions, which classifies pixels according to their spatial proximity, the gradient of their gray levels and the homogeneity of their textures. To avoid over segmentation a single marker for each object of interest has to be selected [17]. The selection of adequate markers on these kinds of images is a painful and sometimes fruitless task. Hence, the experienced observer defines markers in a semiautomatic way [18] [19] [20]. The automatic determination of markers is still a difficult goal to achieve. The current determination algorithms are highly dependent on the structure to be segmented [21] [22]. Moreover, they have a high computational cost and they determine markers in an effective but not automatic way when processing images [23].

The proposed algorithm uses, characteristics determined from the regions resulting from the oversegmentation produced by the watershed transform (WT) through regional minima. WT markers are selected as the cluster that represents the objects of interest. Finally WT is applied again over these new markers allowing an effective and robust segmentation of images. In this way two stage marker controlled watershed method is implemented.

\subsection{Input Images}

\section{Inputs, Methods And Results}

For this work, microscope images were used in order to evaluate the proposed algorithms due to the great difficulty that their segmentation presents. Cardiovascular microscopic images having atherosclerosis were used. In these images we need to segment the microscopic images in order to make a diagnosis. Image resolution is $3150 \times 1446$ pixels, 24 bpp. Figure 2(a) and 3(a) top original image is severe atherosclerosis of the aorta in which the atheromatous plaques have undergone ulceration along with formation of overlying mural thrombus. Figure 2(a) and 3(a) middle original image is coronary atherosclerosis with the complication of hemorrhage into atheromatous plaque, seen here in the center of the photograph. Such hemorrhage acutely may narrow the arterial lumen. Figure 2(a) and 3(a) bottom original image is Berkeley dataset image no. 317080, which is used here for algorithm validation.

\subsection{Inputs and Methods}

The proposed methodology is a two stage process. The first process uses WS to produce a primary segmentation of the input image, while the second process applies the marker controlled watershed segmentation algorithm to the primary segmentation to obtain the final segmentation map. Figure 1 describes block diagram of the proposed method. 


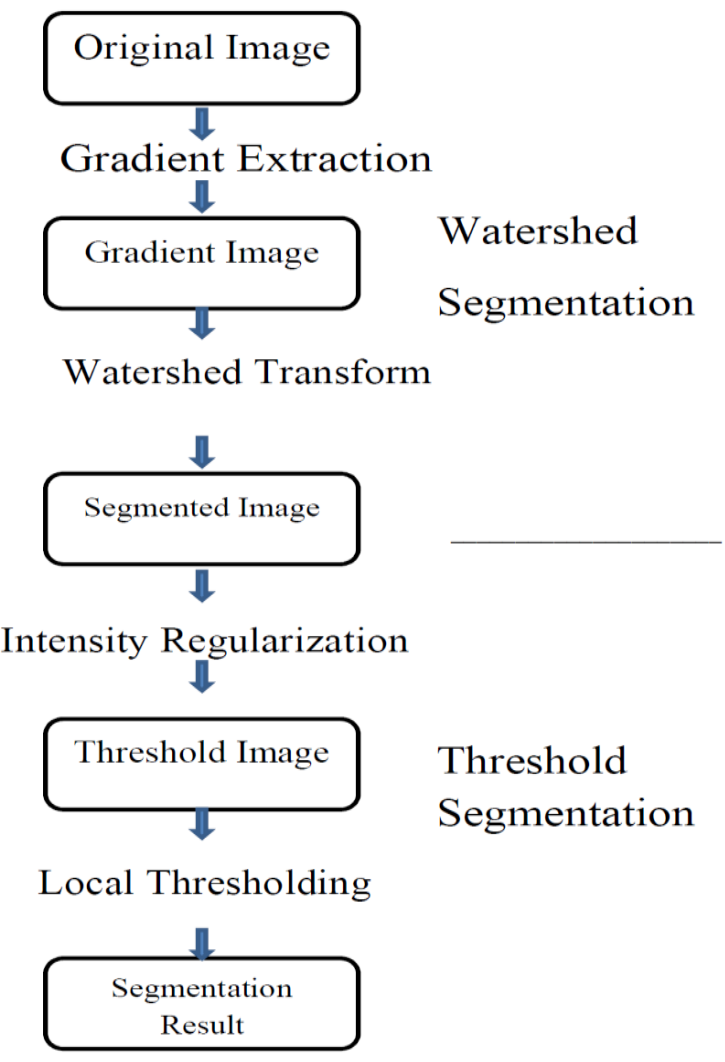

Fig.1 Block diagram of proposed segmentation procedure

\subsubsection{Watershed Transformation Based Segmentation}

1) Watershed Segmentation

Detecting Touching Objects is a challenging problem. Using Watershed Segmentation this problem can be solved. Here we used various morphological functions to perform marker-control watershed segmentation. A potent and flexible method for segmentation of objects with closed contours, where the extremities are expressed as ridges is the Marker-Controlled Watershed Segmentation. In Watershed Segmentation, the Marker Image used is a binary Image comprising of either single marker points or larger marker regions. In this, each connected marker is allocated inside an object of interest. Every specific watershed region has a one-to-one relation with each initial marker; hence the final number of watershed regions determines the number of markers. Post Segmentation, each object is separated from its neighbours as the boundaries of the watershed regions are arranged on the desired ridges. The markers can be manually or automatically selected, automatically generated markers being generally preferred. A solution to limit the number of regional minima is to use markers to specify the only allowed regional minima. The watershed transformation is a powerful tool for image segmentation. The segmentation of images by means of the watershed transform and the use of markers have many advantages: 1] the watershed transform provides closed contours by construction. 2] It avoids severe over-segmentation. In watershed segmentation topographic interpretation, there are 3 types of points: 1) Points belonging to a regional minimum 2) Points at which a drop of water would fall to a single minimum (The catchment basin or watershed of that minimum.) 3) Points at which a drop of water would be equally likely to fall to more than one minimum. (The divide lines or watershed lines.) The elements labelled 1 belong to the first watershed region, the elements labelled 2 belong to the second watershed region, and so on. If the elements labelled 0 do not belong to a unique watershed region. These are called watershed pixels. 8-connected neighbourhoods are used for watershed implementation.

A gray scale image can be interpreted as the topographic image of land relief. It can be indicated that the gray intensities of higher amplitude correspond to plains and mountains and the lower intensity ones correspond to valleys and rivers [24] [25]. Using the characteristics of these images we define a technique of digital image processing called Watershed Transform (WT), which through the flooding of the valleys, is capable of recognizing similar topographical areas, surrounded by mountain ridges. The WT is a segmentation method based on regions, which classifies pixels according to their spatial proximity, the gradient of their gray levels and the homogeneity of their textures [26] [27]. With the objective of segmenting an image in gray levels, prior to the application of the WT, a gradient image must be obtained, where the levels of the contours of the 
objects to be segmented represent an area of elevated gray intensity. The areas of low intensity give way to the basins where the water would flow and flood the topography of the image. The elevations in gray levels generated by the contours would remain and give way to the segmentation of the image through the resulting watershed lines [17] [26]. Mathematical morphology allows us to obtain a gradient which is highly adaptable to different kinds of images with a higher precision than conventional algorithms. In this paper we used the morphological gradient to obtain the intermediate image before applying the WT [28] [29].

The classic WT floods the gradient image from its regional minima. In non-homogeneous or noise embedded images there is not a one to one relation between regional minima and objects of interest. This results in an over segmentation in the majority of images, in other words, after WT each of the objects is represented by more than one region [17-19] [26] [30]. To avoid this over segmentation we resort to the selection of a single marker for each object of interest. Theses markers or seeds initiate the flooding algorithms indicating the sector that gives rise to the basins. Based on these characteristics we can conclude that the success of the WT depends mainly upon the characteristics of the markers.

\subsubsection{Fuzzy C-means algorithm}

Fuzzy C-M clustering [31] (FCM), also called as ISODATA, is a data clustering method in which each data point belongs to a cluster to a degree specified by a membership value. FCM is used in many applications like pattern recognition, classification, and image segmentation. FCM [32] divides a collection of $n$ vectors $\mathrm{c}$ fuzzy groups and finds a cluster centre in each group such that a cost function of dissimilarity measure is minimized. FCM [33] uses fuzzy partitioning such that a given data point can belong to several groups with the degree of belongingness specified by membership values between 0 and 1 . This algorithm is simply an iterated procedure. The algorithm is given below:

1] Initialize the membership matrix $U$ with random values between 0 and 1 .

2] Calculates $C_{\text {fuzzy cluster center }} C_{i}, \mathrm{i}=1,2, \ldots C_{\text {., using the following equation: }}$

$$
C_{i}=\frac{\sum_{j=1}^{n} u_{i j}^{m} x_{j}}{\sum_{j=1}^{n} u_{i j}^{m}}
$$

3] Compute the cost by following equation. Stop when it is below a certain threshold or it improves over previous iteration.

$$
J\left(U, C_{1, \ldots, \ldots} C_{c}\right)=\sum_{i=1}^{c} J_{i}=\sum_{i=1}^{c} \sum_{j}^{n} u_{i j}^{m} d_{i j}^{2}
$$

4] Compute a new $U$ by the equation. Go to step 2 .

$$
u_{i j}=\frac{1}{\sum_{k=1}^{c}\left(\frac{d_{i j}}{d_{k j}}\right)^{\frac{2}{(m-1)}}}
$$

There is no guarantee ensures that FCM [34] converges to an optimum solution. The performance is based on the initial cluster centers. FCM also suffers from the presence of outliers and noise and it is difficult to identify the initial partitions.

\subsubsection{Color Cluster segmentation}

We also implemented color cluster segmentation algorithm. Features extracted from over segmentation resulting regions, after applying WT from its regional minima, are used as input to a clustering algorithm [23] [35]. There are different methods that group these regions to segment images; however none uses them to obtain the markers for the WT [36]. The basis for the development of this algorithm was to reduce the sensitivity of the algorithm to noise and irrelevant objects and to increase its robustness to process medical images with different features. We used two clustering algorithms. First the k means algorithm was used [22] [25] [37]. This unsupervised algorithm requires the specification of the number of classes in which the data set is going to be partitioned. To each class there is a corresponding cluster centre so that the distance of each pattern to its center 
is minimal. The partition is done by measuring, in an iterative process, the distance between each pattern to its cluster center, and computing again the centres until there is no change. In a second stage the fuzzy k-means algorithm was used [21] [25]. This algorithm is based on fuzzy logic and assigns to each pattern a level of belonging to each class instead of offering a unique aggrupation of them.

Different regions are obtained by applying the WT to the original image. The mean and standard deviation of each region characterize different components in the images. The k-means algorithm applied to the features in those regions determined effectively the internal markers of the objects. The fuzzy k-means did not classify correctly the markers of any kind of regions. We applied morphological operators to the class that represents the objects to generate the internal markers. The markers of the background, external markers, were obtained eroding the internal markers complements. This procedure resulted in adequate segmentations when internal markers sizes are similar to the size of the objects. The gradient of the medical images does not have a visible contrast, making it difficult to apply the flooding algorithms. The method based on clustering is malleable and can be applied to all kinds of images. Consequently, by obtaining internal and external markers of a considerable size the results were improved. These properties of the markers were attained due to the use of the algorithm developed from the over segmentation.

\subsubsection{K-means algorithm}

K-means algorithm [31] is under the category of Squared Error-Based Clustering (Vector Quantization) and it is also under the category of crisp clustering or hard clustering. K-means algorithm is very simple and can be easily implemented in solving many practical problems. K-Means is ideally suitable for biomedical image segmentation since the number of clusters $(\mathrm{k})$ is usually known for images of particular regions of human anatomy. Steps of the K-means algorithm are given below:

1) Choose $\mathrm{k}$ cluster centers to coincide with $\mathrm{k}$ randomly chosen patterns inside the hyper volume containing the pattern set $(\mathrm{C})$.

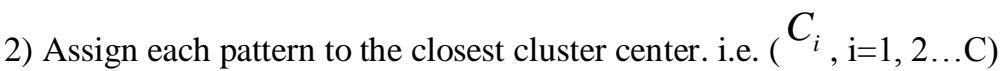

3) Recomputed the cluster centers using the current cluster memberships. (U):

4)

$$
u_{i j}=\left\{\begin{array}{c}
1, i f\left\|x_{j}-c_{i}\right\|^{2} \leq\left\|x_{j}-c_{k}\right\|^{2}, \text { foreach } \neq i \\
0, \text { otherwise }
\end{array}\right\}
$$

5) If convergence criterion is not met, go to step 2 with new cluster centers by the following equation, i.e. minimal decrease in squared error:

6)

$$
c_{i}=\frac{i}{G_{i}} \sum_{k, X_{k} \in G_{i}} X_{k}
$$

$$
\text { Where, }\left|G_{i}\right|_{\text {is the size of }} G_{i} \text { or }\left|G_{i}\right|=\sum_{j=1}^{n} u_{i j}
$$

The performance of the K-means algorithm depends on the initial positions of the cluster centers.

This is an inherently iterative algorithm. And also there is no guarantee about the convergence towards an optimum solution. The convergence centroids vary with different initial points. It is also sensitive to noise and outliers. It is only based on numerical variables. Coronary atherosclerosis images were processed with a clustering algorithm. Figure 2(e) shows the images resulting from the application of the clustering algorithm and figure 3(e) shows corresponding cluster plots. The Atherosclerosis images successful segmentation can be seen.

\subsubsection{Watershed Transform based algorithm:}

Step 1 Application of the WT using the regional minima as markers. The result is a matrix that assigns a label to each pixel indicating the regional minima to which it belongs.

Step 2 The regions resulting from the WT are analysed to obtain features from each one of them to distinguish the objects of interest based on their texture. Mean and standard deviation are computed from the intensities that are found inside the regions.

Step 3 The features vectors are used as input for the k-means algorithm.

Step 4 An image is obtained with only the class that distinguishes the objects of interest.

Step 5 Application of the morphological opening and closing operators on the markers to obtain the object markers, or internal markers [28] [29]. These operators join adjacent regions because they correspond 
to the same object and eliminate regions that do not belong to the wanted objects. The previous result is slightly eroded to eliminate the regions that belong to the possible borders of the objects. As a final result we obtain the image that is to be used as the internal marker for the WT.

Step 6 Application of the morphological erosion to the complement of the internal markers, to obtain the background markers, or external markers.

Step 7 Application of WT using the internal and external markers computed in the previous steps, over the morphological gradient of the original image.

\subsubsection{Proposed Marker Controlled Watershed Segmentation algorithm:}

Segmentation using the watershed transforms works well if you can identify, or mark, foreground objects and background locations. The gradient magnitude of the primary segmentation is obtained by applying the Sobel operator. The Canny edge detector was also experimented on, but it was found that the results obtained by both methods are comparable. Hence, we decided on the Sobel filter as the Canny edge detector has higher complexity. In addition, the Sobel filter has the advantage of providing both a differencing and smoothing effect. Marker controlled watershed segmentation follows this basic procedure:

1) Compute a segmentation function. This is an image whose dark regions are the objects we are trying to segment.

2) Compute foreground markers. These are connected blobs of pixels within each of the objects.

3) Compute background markers. These are pixels that are not part of any object.

4) Modify the segmentation function so that it only has minima at the foreground and background marker locations.

5) Compute the watershed transform of the modified segmentation function. Finally the WT was applied to the gradient image of the original image using the markers obtained in the previous steps. It was not possible to obtain this result with other conventional methods of image segmentation.

Openings with structuring elements of $3 \times 3$ pixels were applied, obtaining unequivocal and homogeneous internal markers for each one of the objects of interest. To apply the WT it is necessary to mark not only the objects but also the background. We need to define the internal markers for the objects of interest as well as the external markers. The latter ones were obtained through the morphological erosion of the complement of the internal markers. To obtain the object markers, or internal markers, morphological opening and closing operators are applied. These unite the adjacent regions that correspond to the same object and eliminate the regions that do not belong to the wanted objects. Then the result is eroded to eliminate the regions that belong to the possible borders of the objects. As a result the internal markers for the WT are obtained. The background markers or external markers are obtained eroding the complement to the internal markers. Finally, the WT is again calculated with the internal and external markers previously determined and the morphological gradient of the original image. Figure 3(c) shows the resulting segmentation. Medical images were successfully segmented. It was possible to analyse atherosclerosis images that could not be segmented with other algorithms of marker detection. The morphological operations and WT have a minimal computational cost. The error of the algorithms can be determined by a parameter $\mu$ based on the symmetrical distance and the Hausdorff distance [38].

Segmentation using the watershed transforms works well if we can identify, or "mark," foreground objects and background locations. Marker-controlled watershed segmentation (figure 6) follows this basic procedure:

Step 1: Read in the original color microscopic images of coronary artery and convert it to grayscale.

Step 2: Use the gradient magnitude as the segmentation function. Compute a segmentation function. Input various microscopic images whose regions are the objects to be segmented. The gradient is high at the borders

of the objects and low (mostly) inside the objects. Gradient can be computed by $\operatorname{Gradmag}=\sqrt{I_{x}^{2}+I_{y}^{2}}$. The watershed transform directly on the gradient magnitude will generate "over segmentation", so markers are introduced in the image.

Step 3: Mark the foreground objects. These are connected blobs of pixels within each of the objects. The foreground markers, which must be connected blobs of pixels inside each of the foreground objects. Here we used morphological techniques called "opening-by-reconstruction" and "closing-by-reconstruction". These operations will create flat maxima inside each object. Opening is erosion followed by dilation, while openingby-reconstruction is erosion followed by a morphological reconstruction. To obtain good foreground markers, reconstruction-based opening and closing are more effective than standard opening and closing at removing small imperfections without affecting the overall shapes of the objects, for that compute the regional maxima. Ideally we don't want the background markers to be too close to the edges of the objects we are trying to segment. We'll "thin" the background by computing the "skeleton by influence zones". This can be done by 
computing the watershed transform of the distance transform of black and white image, and then looking for the watershed ridge lines (ideally zero) of the result.

Step 4: Compute background markers. These are pixels that are not part of any object. The markers of the background or external markers were obtained by morphological erosion of the internal markers complements. This procedure resulted in adequate segmentations when the internal markers and the objects of interest are of similar size. Perform minima imposition through morphological reconstruction by erosion of $\left.\left(f_{g}+1\right) \wedge f_{m}\right)$ from $f_{m}$ which can be written by $f_{g \min }=R_{\left(f_{g}+1\right) \wedge f_{m}}^{E}\left(f_{m}\right)$ so that markers are only at the single minima (figure 1(a)). Thus we obtain automatic selection of markers. Where, $f_{g}=$ gradient image having many local minima; $f_{m}=$ marker image; $R^{E}=$ Region created through morphological reconstruction by erosion; $f_{g \text { min }}=$ single global minimum morphological reconstruction.

Step 5: Modify the segmentation function so that it only has minima at the foreground and background marker locations. Here, modify the gradient magnitude image, so that its only regional minima occur at foreground and background marker pixels.

Step 6: Compute the watershed transform (second time) of the modified segmentation function. In this application on microscopic images of coronary arteries, we have applied two stage watershed algorithms to take care for over segmentation.

Step 7: Obtained threshold image. Here threshold selected for the skeleton of the image.

Step 8: Representation and Visualization of the results in form of color watershed. For better interpretation of results, one visualization technique is to superimpose the foreground markers, background markers, and segmented object boundaries on the original image.

Step 9: To help interpret the result, superimpose the color segmented image on the original image.

The intuitive idea underlying the notion of watershed comes from the field of topography: a drop of water falling down on a topographic surface follows a descending path and reaches a regional minimum area. The watershed may be thought of as the separating lines of the domain of attraction of drops of water.

The regions of a watershed, also called catchment basins, are associated with the regional minima of the map. In other words, each catchment basin contains a unique regional minimum, and conversely, each regional minimum is included in a unique catchment basin: the regions of the watershed are the connected components of an extension relative to the minima. They are separated by a set of edges from which a drop of water can flow down towards different minima.

\subsubsection{Assessment parameters:}

A] The structural similarity (SSIM) index [39] is a method for measuring the similarity between two images. The SSIM index is a full reference metric, in other words, the measuring of image quality based on an initial uncompressed or distortion-free image as reference. SSIM is designed to improve on traditional methods like peak signal-to-noise ratio (PSNR) and mean squared error (MSE), which have proved to be inconsistent with human eye perception.

The SSIM metric is calculated on various windows of an image. The measure between two windows $x$ and $y$ of common size $N \times N$ is:

$$
\operatorname{SSIM}(x, y)=\frac{\left(2 \mu_{x} \mu_{y}+c_{1}\right)\left(2 \sigma_{x y}+c_{2}\right)}{\left.\mu_{x}^{2}+\mu_{y}^{2}+c_{1}\right)\left(\sigma_{x}^{2}+\sigma_{y}^{2}+c_{2}\right)}
$$

With

$\mu_{x}$ The average of $x$;

$\mu_{y}$ The average of ${ }^{y}$;

$\sigma_{x}^{2}$ The variance of $x$;

$\sigma_{y}^{2}$ The variance of $y$;

$\sigma_{x y}$ The covariance of $x_{\text {and }} y$;

$c_{1}=\left(k_{1} L\right)^{2}, c_{2}=\left(k_{2} L\right)^{2}$ two variables to stabilize the division with weak denominator; 
$L$ The dynamic range of the pixel-values (typically this is $2^{\# \text { bitsperpixel }}-1$ ); $k_{1}=0.01$ and $k_{2}=0.03$ by default.

In order to evaluate the image quality this formula is applied only on luma. The resultant SSIM index is a decimal value between -1 and 1 , and value 1 is only reachable in the case of two identical sets of data.

B] The universal image quality index (UIQI or Q) which measures the distortion between two images. The dynamic range of $\mathrm{Q}$ is between -1 and 1 , the best value of $\mathrm{Q}=1$ is achieved if and only if the two images are equal. Higher the $\mathrm{Q}$ values, the less will be the distortion between the original and the processed image.

\section{Results and Discussion}

We implemented these algorithms on a 64 bit operating system Window 7(Ultimate), Mat Lab 7.11.0 (R2010b) [40] (Intel Core i5 3.6 GHz and 4GB RAM). In this research paper, we compared results of Otsu thresholding, Fuzzy C-means clustering, K-means clustering and marker controlled watershed algorithm. And also we showed that our proposed method produced segmentation maps which gave fewer partitions than the segmentation maps produced by the conservative watershed algorithm.
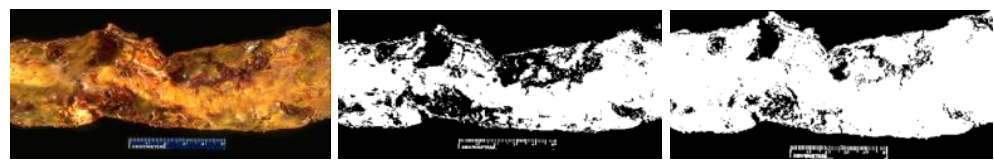

Threshold level $\mathrm{T}=0.356863$
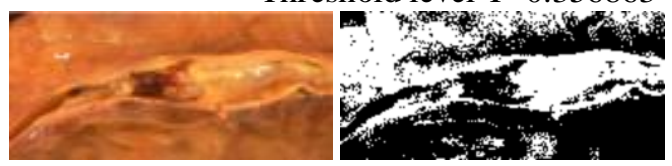

$\mathrm{T}=0.2333$

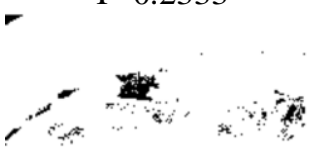

$\mathrm{T}=0.521569$

(a)

(b)

$\mathrm{T}=0.319608$

(c)
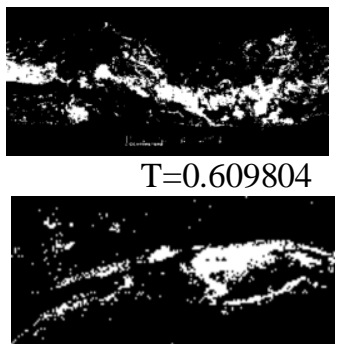

$\mathrm{T}=0.641176$

(d)

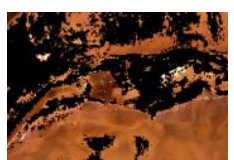

(e)

Fig. 2 Results of Otsu, FCM0 level, FCM1 level and Color Clustering (CC) Segmentation Techniques (a) Original images of Atherosclerosis, Coronary Atherosclerosis (b) Results of Otsu Thresholding method (c) Results of Fuzzy C Means level 0 method (d) Results of Fuzzy C Means level 1 method (e) Segmentation result of CC technique.
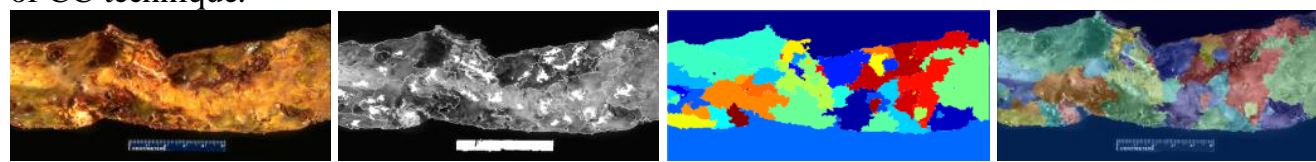

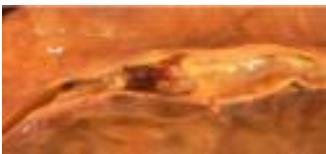

(a)

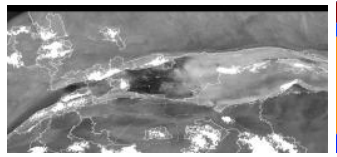

(b)

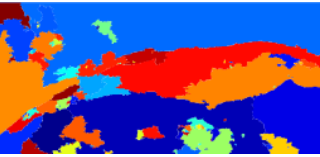

(c)

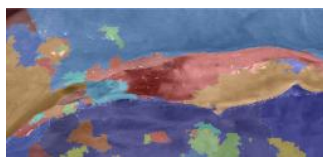

(d)

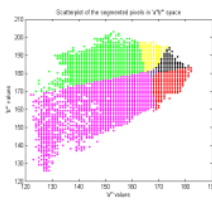

(e) images of Atherosclerosis, Coronary Atherosclerosis (b) Gradient magnitude of original image of figure 3(a) with MCWS (c) Colored segmentation using MCWS (d) Colored segmentation superimposed on original image of figure 3(a) (e) Graph using CC technique.

TABLE 1: Formulas for assessment parameters

\begin{tabular}{|l|c|c|}
\hline $\begin{array}{l}\text { Name of } \\
\text { Parameters }\end{array}$ & Formulas & $\begin{array}{l}\text { Ideal or Desired } \\
\text { Value }\end{array}$ \\
\hline Mean Square Error & $M S E=\frac{1}{M N} \sum_{m=1}^{M} \sum_{n=1}^{N}\left(x_{m, n}-\bar{x}_{m, n}\right)^{2}$ & Lower \\
\hline
\end{tabular}




\begin{tabular}{|l|l|l|}
\hline $\begin{array}{l}\text { Peak Signal to } \\
\text { Noise Ratio }\end{array}$ & $P S N R=10 \log \frac{\left(2^{n}-1\right)^{2}}{M S E}=10 \log \frac{255^{2}}{M S E}$ & Higher \\
\hline $\begin{array}{l}\text { Normalized } \\
\text { Absolute Error }\end{array}$ & $N A E=\sum_{m=1}^{M} \sum_{n=1}^{N}\left|x_{m, n}-\bar{x}_{m, n}\right| / \sum_{m=1}^{M} \sum_{n=1}^{N}\left|x_{m, n}\right|$ & Lower \\
\hline
\end{tabular}

TABLE 2: Results of segmented image of Atherosclerosis figure 3(c) upper with assessment parameters

\begin{tabular}{|c|c|c|c|c|c|c|c|c|c|}
\hline $\begin{array}{c}\text { Sr. } \\
\text { No. }\end{array}$ & Methods & MSE & PSNR & NAE & STD & CoV & UIQI & $\begin{array}{c}\text { Corr } \\
\text { Coeff }\end{array}$ & SSIM \\
\hline 1 & MCWS & $\mathbf{5 7 0 9 . 3}$ & $\mathbf{1 0 . 5 6 5 0}$ & 0.8573 & $\mathbf{7 1 . 0 3 6 1}$ & $\mathbf{2 2 0 7 . 9}$ & 0.4539 & 0.4876 & 0.4570 \\
\hline 2 & Otsu & 6204.9 & 10.2034 & 0.8135 & 120.7704 & 6764.3 & $\mathbf{0 . 6 7 7 0}$ & $\mathbf{0 . 8 7 9 2}$ & $\mathbf{0 . 6 7 7 8}$ \\
\hline 3 & FCM0 & 15290 & 6.2867 & 1.3402 & 119.8344 & 5236 & 0.4299 & 0.6859 & 0.4309 \\
\hline 4 & FCM1 & $\mathbf{5 5 4 9}$ & $\mathbf{1 0 . 6 8 8 7}$ & $\mathbf{0 . 7 7 8 4}$ & 79.2966 & 3187.4 & 0.4607 & 0.6310 & 0.4624 \\
\hline
\end{tabular}

TABLE 3: Results of segmented image of Coronary atherosclerosis figure 3(c) lower with assessment

\begin{tabular}{|c|c|c|c|c|c|c|c|c|c|}
\hline $\begin{array}{c}\text { Sr. } \\
\text { No. }\end{array}$ & Methods & MSE & PSNR & NAE & STD & CoV & UIQI & $\begin{array}{c}\text { Corr } \\
\text { Coeff }\end{array}$ & SSIM \\
\hline 1 & MCWS & $\mathbf{4 2 5 3 . 4}$ & $\mathbf{1 1 . 8 4 3 4}$ & $\mathbf{0 . 4 3 8 9}$ & $\mathbf{5 3 . 8 7 6 5}$ & 537.5480 & 0.2683 & 0.3435 & 0.2785 \\
\hline 2 & CCT & 9632.1 & 8.2936 & 0.5338 & 58.7586 & -430.4538 & -0.1560 & -0.2522 & -0.1435 \\
\hline 3 & Otsu & 9363.1 & 8.4166 & 0.7129 & 114.3957 & 2430.9 & $\mathbf{0 . 3 4 5 5}$ & $\mathbf{0 . 7 3 2 4}$ & $\mathbf{0 . 3 4 8 2}$ \\
\hline 4 & FCM0 & 15604 & 6.1984 & 0.9415 & $\mathbf{4 2 . 7 0 4 8}$ & $\mathbf{3 4 6 . 7 1 9 4}$ & 0.2139 & 0.2798 & 0.2269 \\
\hline 5 & FCM1 & 14703 & 6.4567 & 0.9037 & 71.2572 & 602.0788 & 0.0860 & 0.2912 & 0.0894 \\
\hline
\end{tabular}

Abbreviations: MCWS=Marker Control Watershed Segmentation method; Otsu= Otsu Thresholding method; CCT=Color Clustering Technique; FCM0=FCM 0 level method; FCM1=FCM 1 level method; MSE=Mean Square Error; PSNR=Peak Signal to Noise Ratio; NCC=Normalized Cross Correlation; $A D=$ Average Difference; $\mathrm{SC}=$ Structural Content; NAE $=$ Normalized Absolute Error; STD=Standard Deviation; $\mathrm{CoV}=$ Covariance; UIQI=Universal Image Quality Index; Corr Coeff = Correlation Coefficient; SSI=Structural Similarity Index Matrix.

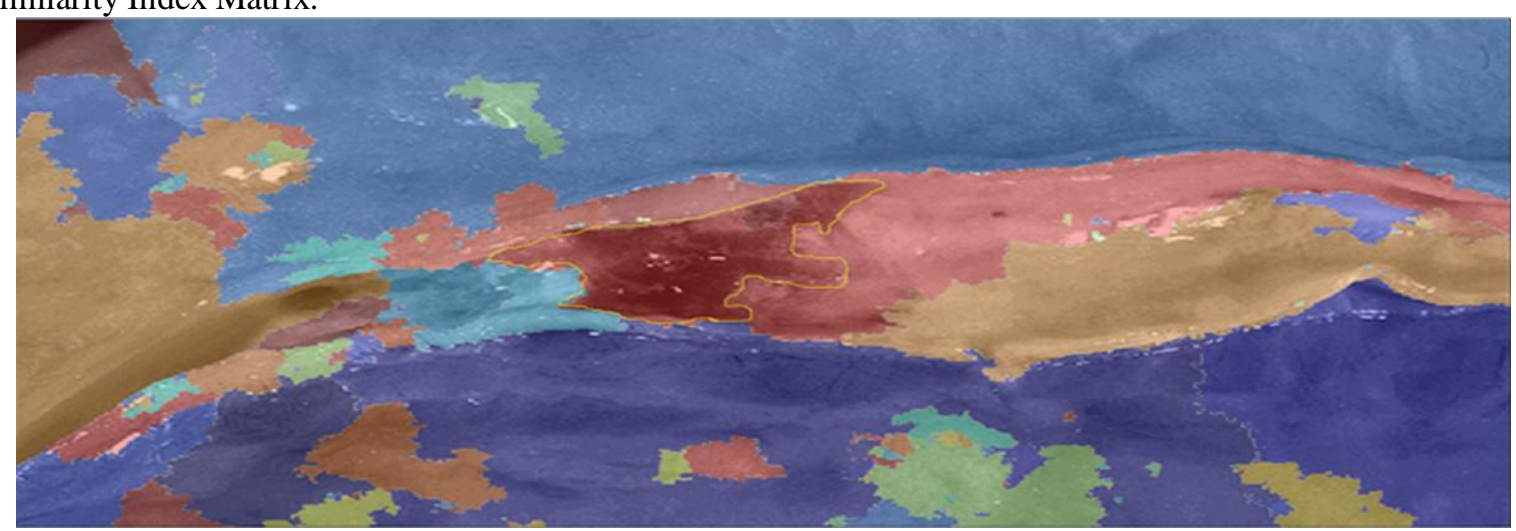

Figure 4 Region of interest of segmented image with proposed technique for coronary atherosclerosis with the complication of hemorrhage into atheromatous plaque. 


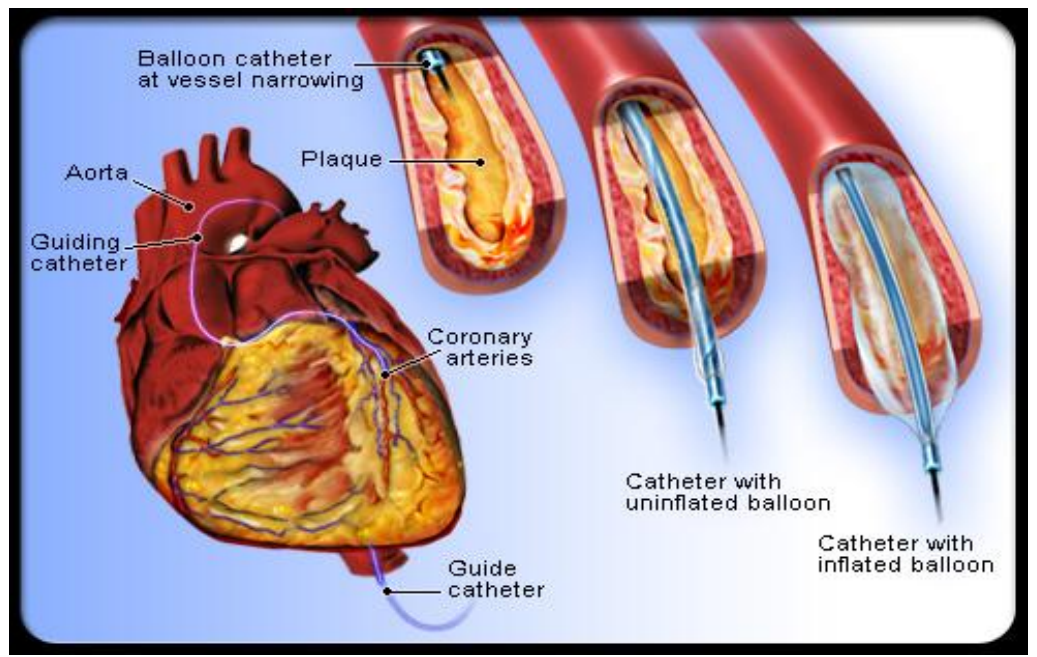

Figure 5: Insertion of Catheter in coronary arteries to remove plaque [41].

We applied our proposed methodologies of K Means clustering, Fuzzy C-means clustering and marker controlled watershed algorithm to medical images of the atherosclerosis images and obtained general segmentation maps of them. Fig. 2 shows the results of Otsu, FCM0 level, FCM1 level and Color Clustering (CC) Segmentation Techniques. Fig. 2 (a) is the original images of Atherosclerosis of aorta and Coronary Atherosclerosis. Fig. 2 (b-e) shows the results of Otsu thresholding method, results of fuzzy $\mathrm{C}$ means level 0 methods, and results of fuzzy $\mathrm{C}$ means level 1 method and segmentation result of $\mathrm{CC}$ technique respectively. Otsu, FCM0 level, FCM1 level and Color Clustering (CC) Segmentation Techniques are considered for comparisons because those methods provide segmentation results which are visually and quantitative way comparable with our proposed segmentation technique MCWS.
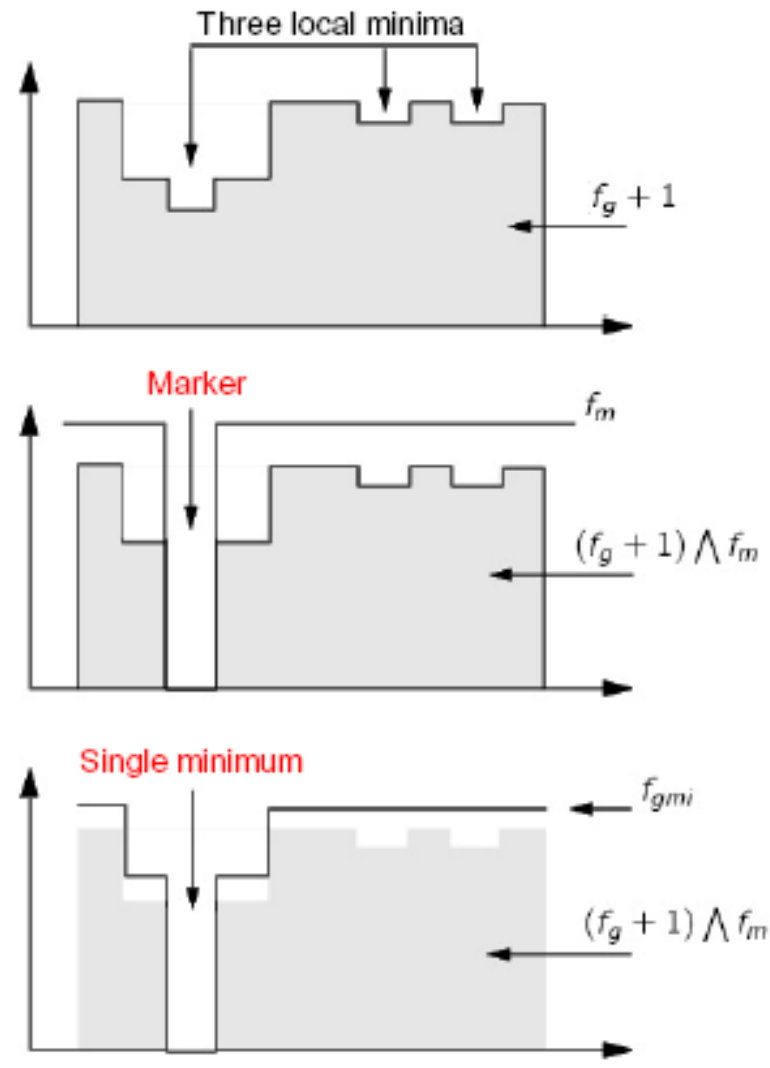

Figure 6: Representation of proposed watershed transformation in two dimensions 
TABLE 4: Values for Region of Interest of segmented image of figure 4

\begin{tabular}{|c|c|c|c|c|c|c|c|c|c|}
\hline Image & Area & Mean & StdDev & Perim. & Circ. & Feret & IntDen & Skew & Kurt \\
\hline $\begin{array}{c}\text { Fig } \\
\text { 3(d) }\end{array}$ & 1.7 & 86.67 & 25.31 & 8.524 & 0.3 & 2.9 & 153.0 & $\begin{array}{l}0.65 \\
2\end{array}$ & $\begin{array}{l}1.17 \\
\text { lower }\end{array}$ \\
\hline
\end{tabular}

Abbreviations: StdDev=Standard Deviation; Perim. =Perimeter; Circ=Circularity; IntDen=Integrated Density; Skew=Skewness; Kurt= Kurtosis.

Fig. 3 shows the results of proposed marker control Watershed (MCWS) Segmentation Technique. Fig. 3 (a) is the original images of Atherosclerosis of aorta and Coronary Atherosclerosis. Fig. 3 (b-e) represent the gradient magnitude of original image of figure 3(a) with MCWS , colored segmentation using MCWS , colored segmentation superimposed on original image of figure 3(a) and Graph using CC technique respectively. Table 1 represents the formulas for assessment parameters and mention about what should be their ideal values.

Table 2, 3 and 4 show the segmentation results. Ideally error should be small. So MSE and NAE values should be smaller, which are obtained through proposed MCWS algorithm. A higher value of PSNR is better, which is also obtained by proposed algorithm. Standard deviation and Co variance should be lower value, which is also good for proposed algorithm. As seen from table 2, for Atherosclerosis image using marker controlled WS(MCWS) method, the values of MSE=5709.3, PSNR=10.5650, STD=71.0361 and CoV=2207.9 which are best as compared to Otsu method and Fuzzy C means. It can be observed in table 2, that the values of UIQI=

0.6770, Corr Coeff $=0.8792$ and SSI $=0.6778$ parameters are best for atherosclerosis image using Otsu method among all methods. As seen from table 3, for Coronary Atherosclerosis image using marker controlled WS(MCWS) method, the values of $\mathrm{MSE}=4253.4, \mathrm{PSNR}=11.8434, \mathrm{NAE}=0.4389$ and $\mathrm{STD}=53.8765$, which are best as compared to Otsu method and Fuzzy C means. Results of Coronary atherosclerosis of FCM0 method for $\mathrm{STD}=42.7048$ which is on gray image, while MCWS results are on color images. As seen from table 2 and table 3 , the values of UIQI (range -1 to 1 ) and SSI(range -1 to 1) are better for Otsu thresholding technique for all images i.e. atherosclerosis, coronary atherosclerosis, which proves the consistency of all algorithms. Values toward 1 are better. Here, it is observed that MCWS algorithm gives results in form of color segmentation and provides better results for majority of assessment parameters. TABLE 4 represents the values for Region of Interest (ROI) of segmented image of figure 3(d) lower image, which is coronary atherosclerosis with the complication of hemorrhage into atheromatous plaque. We evaluated the performance of our proposed methodologies by comparing them with conservative watershed algorithm. The use of marker controlled watershed segmentation algorithm had achieved the objective of reducing the problem of over segmentation when applied to atherosclerosis images.

\section{Conclusion}

This paper presents, application of Segmentation Techniques on Atherosclerosis Images using Otsu thresholding method, Fuzzy C means level 0, Fuzzy C means level 1, color clustering algorithm and with marker controlled watershed algorithm. Marker controlled watershed algorithm gave better segmentation than all other algorithms. By reducing the amount of over segmentation, we obtained a segmentation map which is more diplomats of the several anatomies in the medical images. It addressed the limitations of the conservative watershed algorithm, which included over segmentation. Comparing with the algorithm based on fuzzy logic, clustering is not only automatic but it is also able to segment the atherosclerosis images with a lower error. The marker controlled watershed algorithm presents smaller computational costs than the algorithm based on clustering. With our proposed algorithm we obtained desired values for various assessment parameters. Here with proposed algorithm, we obtained MSE and NAE values lower, PSNR values higher. Deviation and Variance of pixels for segmentation results are less. UIQI and SSIM values are sufficiently moderate. Finally, values for Region of Interest of resultant segmented image using proposed MCWS method are computed and presented in table 4 from which severity of atherosclerosis can be determine.

\section{References}

[1] Milan Sonka, Vaclav Hlavac, Roger Boyle, Image Processing, Analysis and Machine Vision, 2nd ed., Bangalore, by Thomson Asia Pte Ltd., Singapore, first reprint 2001, pp-186.

[2] Anil k. Jain, Fundamentals of Digital Image Processing, Delhi, Published by Pearson Education, 3rd Impression, 2008 , pp-370.

[3] J. L. Vincent, "Morphological grayscale reconstruction in image analysis: Application and efficient algorithms," IEEE Trans. Image Proc., vol. 2, pp. 176-201, 1993.

[4] S. Beucher, "Watershed, hierarchical segmentation and waterfall algorithm," Mathematical Morphology and Its Applications to Image Processing, Dordrecht, and The Netherlands: Kluwer pp. 69-76, 1994.

[5] V. Grau, A. U. J. Mewes, M. Alcaniz, R. Kikinis and S. K. Warfield, "Improved Watershed Transform for Medical Image Segmentation Using Prior Information," IEEE Trans. Medical imaging, vol. 23, No. 4, pp. 447-458, April 2004.

[6] Valerie Pazos, Rosaire Mongrain, and Jean-Claude Tardif, "Mechanical Characterization of Atherosclerotic Arteries Using FiniteElement Modeling: Feasibility Study on Mock Arteries,” IEEE Trans. on biomedical Eng., vol. 57, no. 6, June 2010. 
[7] Christof Karmonik, Pamela Basto, Kasey Vickers, Kirt Martin, Micheal J. Reardon, Gerald M. Lawrie, and Joel D. Morrisett, "Quantitative Segmentation of Principal Carotid Atherosclerotic Lesion Components by Feature Space Analysis Based on Multicontrast MRI at 1.5 T," IEEE Transactions on biomedical engineering, vol. 56, no. 2, February 2009.

[8] Danijela Vukadinovic, Theo van Walsum, Rashindra Manniesing, Sietske Rozie, Reinhard Hameeteman, Thomas T. de Weert, Aad van der Lugt, and Wiro J. Niessen, "Segmentation of the Outer Vessel Wall of the Common Carotid Artery in CTA," IEEE Transactions on medical imaging, vol. 29, no. 1, January 2010

[9] Gozde Unal, Susann Bucher, Stephane Carlier, Greg Slabaugh, Tong Fang, and Kaoru Tanaka, "Shape-Driven Segmentation of the Arterial Wall in Intravascular Ultrasound Images," IEEE Trans. on info. Tech. in biomedicine, vol. 12, no. 3, May 2008

[10] Simon Le Floch, Jacques Ohayon, Philippe Tracqui, Gerard Finet, Ahmed M. Gharib, Roch L. Maurice, Guy Cloutier, and Roderic I. Pettigrew, "Vulnerable Atherosclerotic Plaque Elasticity Reconstruction Based on a Segmentation-Driven Optimization Procedure Using Strain Measurements: Theoretical Framework," IEEE Trans. on medical imaging, vol. 28, no. 7, July 2009.

[11] Gonzalez, R.C., R.E. Woods and S.L. Eddins, 2004.Digital Image Processing using MATLAB. ${ }^{\text {st }}$ Edn., Pearson Education India, India, and ISBN: 9788177588989 , pp.: 620.

[12] Jayaraman, S., 2009. 'Digital Image Processing', $1^{\text {st }}$ Edn., Tata McGraw Hill Education, New Delhi, and ISBN: 780070144798, pp: 723.

[13] Murugavalli, S. and V. Rajamani, "An improved implementation of brain tumor detection using segmentation based on neuro fuzzy technique,” J. Comput. Sci., 3: 841-846. DOI: 10.3844/jcssp.2007.841.846, 2007.

[14] Shanmugam, N., A.B. Suryanarayana, D. Chandrashekar and C.N. Manjunath, 2011, "A novel approach to medical image segmentation,”J. Comput. Sci., 7: 657-663. DOI: 10.3844/jcssp.2011.657.663

[15] Velmurugan, T. and T. Santhanam, "Computational complexity between k-means and kmedoids clustering algorithms for normal and uniform distributions of data points" J. Comput. Sci., 6: 363-368. DOI: 10.3844/jcssp.2010.363.368

[16] C. A. Glasbey and G W Horgan, "Image analysis for the biological science," Statistics in Practice, Series Editor Vic Barnett, Jonh Wiley and Sons 1994.

[17] L Vincent and P. Soille, "Watersheds in digital spaces: An efficient algorithm based on immersion simulations," IEEE trans. Pattern Anal, Machine Intell 1991, 13:583-598.

[18] J. M. Gauch, "Image segmentation and analysis via multiscale gradient watershed hierarchies," IEEE Trans. on Image Processing 1999, 8 (1):69-79

[19] P. Jackway, "Gradient Watersheds in morphological scalespace," IEEE trans. Image Processing, Machine Intell., 1996, 5:913-921

[20] D. Wang, "A multiscale gradient algorithm for Image Segmentation using Watersheds," Pattern Reconition, 1997, 30:2043-2052

[21] P. V. Gorsevski, P. E. Gessler and P. Jankowski, "Integrating a fuzzy k-means classification and bayesian approach for spatial prediction of landslide hazard," J. of Geographical Systems, 2003, 5:5930-5949.

[22] X. Li and G. Harnarneh, "Modeling prior shape and appearance knowledge in watershed segmentation," Image Vision Comput, 2007.

[23] M. A. Gonzalez and V. L. Ballarin, "Determinación automática de marcadores para la Transformada Watershed mediante técnicas de clasificación de patrones," 9vo Simposio Argentino de Informática y Salud (SIS), 2006

[24] K.Castleman, Digital Image Processing. Ch. 15, Prentice Hall 1979

[25] R. González and R. Woods, Addison Wesley 1996.

[26] S. Beucher and F. Meyer, "The morphological approach to segmentation: The Watershed Transformation," Mathematical Morphology and its application to image processing, optical engineering. TT16 1994

[27] M. Couprie and G. Bertrant, "Topological grayscale Watershed Transform," SPIE Vision Geometry Proceedings 1997, 3168:136146

[28] E. R. Dougherty J. and Astola, “An introduction to nonlinear image processing,” Ed. New York: Marcel Decker 1993, 433-481

[29] Mariela A. Gonzalez, Teresita R. Cuadrado, Virginia L. Ballarin, "Comparing marker definition algorithms for Watershed segmentation in microscopy images," JCS\&T Vol. 8 No. 3 October 2008

[30] L. S. Stanislav and S. Wolfgang, "Extracting regions of interest applying a local watershed transformation," Proc. of IEEE Visualization 2000

[31] M.C. Jobin Christ and R.M.S. Parvathi, "Segmentation of Medical Image using Clustering and Watershed Algorithms," American Journal of Applied Sciences 8 (12): 1349-1352, 2011 ISSN 1546-9239

[32] M. Ceccarelli, F. Musacchia and A. Petrosino, "A fuzzy scale-space approach to feature-based image representation and retrieval," Brain Vision and Artificial Intelligence 2006, 377-385

[33] Ceccarelli M and Petrosino A, "A parallel fuzzy scale-space approach to the unsupervised texture separation," Pattern Recognition Letters 2002, 5: 557-567.

[34] Cheng H D, Chen Y H and Sun Y, “A novel fuzzy entropy approach to image enhancement and thresholding," Signal Processing, 1999, 75:277-301.

[35] Gonzalez M A and Ballarin V L, "Segmentación de imagines mediante la Transformada Watershed: Obtención de marcadores mediante Morfología Matemática," 9vo Simposio Argentino de Informática y Salud (SIS), 2006

[36] S. E. Hernandez and K. E. Barner, "Joint region merging criteria for Watershed-Based image segmentation," IEEE Trans on Image Processing, 2000

[37] J. B. Mc Queen, "Some Methods for Classification and Analysis of Multivariate Observation," Proceedings of the $5^{\text {th }}$ Berkeley Symposium on Mathematical Statistics and Probability 1967, Berkeley University of California Press, 281-297.

[38] J.Pastore, E.Moler and V.Ballarin, "Comparison of segmentation techniques by means of non-Euclidean distances," Digital Signal Processing (revision) 2006.

[39] http://en.wikipedia.org/wiki/Structural_similarity.

[40] http://www.mathworks.in

[41] http://saintlukeshealthsystem.adam.com/content.aspx?productId=108\&pid=1\&gid=000171

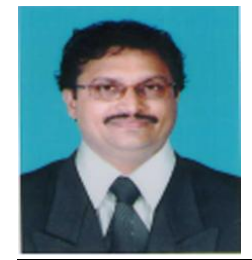

Janak B. Patel (born in 1971) received B.E. (Electronics \& Communication Engg from L.D. College of Engg. Ahmedabad, affiliated with Gujarat University and M.E. (Electronics Communication \& System Engg.) in 2000 from Dharmsinh Desai Institute of Technology, Nadiad, affiliated with Gujarat University. He is Asst. Prof. \& H.O.D. at L.D.R.P. Institute of Technology \& Research, Gandhinagar, and Gujarat. He has submitted his Ph.D. thesis at Indian 
Institute of Technology, Roorkee under quality improvement program of AICTE, India. His research interest includes digital signal processing, image processing, bio-medical signal and image processing. He has 7 years of industrial and 12 years of teaching experience at Engineering College. He taught many subjects in EC, CS and IT disciplines. He is a life member of CSI, ISTE \& IETE.

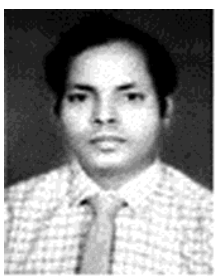

R.S. Anand received B.E., M.E. and Ph.D. in Electrical Engg. from University of Roorkee (Indian Institute of Technology, Roorkee) in 1985, 1987 and 1992, respectively. He is a professor at Indian Institute of Technology, Roorkee. He has published more than 100 research papers in the area of image processing and signal processing. He has also supervised $10 \mathrm{PhDs}, 60 \mathrm{M}$.Tech.s and also organized conferences and workshops. His research areas are biomedical signals and image processing and ultrasonic application in non-destructive evaluation and medical diagnosis. He is a life member of Ultrasonic Society of India. 